\title{
TRENDS IN LABORATORY DIAGNOSTIC METHODS IN PERIODONTOLOGY
}

\author{
Beáta Bolerázska', Mária Mareková ${ }^{1}$, Neda Markovská ${ }^{2}$
}

Department of Medical and Clinical Biochemistry, Faculty of Medicine, P. J. Šafárik University in Košice, Košice, Slovakia ${ }^{1}$; 1st Department of Stomatology, Louis Pasteur University Hospital, Košice, Slovakia²

Summary: This work presents a summary of current knowledge on the laboratory diagnosis of periodontitis. It focuses on the theoretical foundations and is supplemented with new knowledge. It subsequently describes specifically the laboratory diagnosis methods of periodontitis: the protein expression of inflammation, oral microbiology and molecular diagnostics. Periodontitis is a serious disease worldwide and its confirmed association with systemic diseases means its severity is increasing. Its laboratory diagnosis has the potential to rise to the level of clinical and diagnostic imaging. The transfer of diagnostic methods from laboratory to clinical use is increasingly used in the prevention and monitoring of the exacerbation and treatment of periodontal disease, as well as of its impact on systemic disease.

Keywords: Periodontitis; Diagnosis; Biomarkers; Saliva; Gingival crevicular fluid; Point-of care testing; Markers of bone remodelling

\section{Introduction}

Periodontitis is generally defined as an inflammatory disease of the supporting tissues of the tooth, caused especially by a particular microorganism or group of microorganisms, resulting in progressive destruction of the supporting tissues of the tooth - the periodontal tissue. The undeniable role of bacterial infection in the pathogenesis of the disease is now known to be accompanied by the individual's immune and inflammatory response under the influence of external (e.g. dental plaque) and internal factors (genetic makeup of the individual).

It is believed that over $50 \%$ of the European population suffers from various forms of periodontitis, and in more than $10 \%$ this condition is serious. The population of those 60 to 65 years old has a prevalence that runs as high as $70-85 \%$ (24). Periodontal disease appears to be more common in men than in women (44). It has repeatedly been demonstrated that especially periodontitis, may affect the course of a number of systemic diseases, such as coronary heart disease and stroke, diabetes mellitus, osteoporosis, respiratory diseases and also increases risk of low birth weight (31). The threats posed by periodontal diseases to individuals with chronic diseases is caused by three principal mechanism: (i) metastatic spread of infection from the oral cavity as a result of transient bacteremia, (ii) metastatic injury from the effects of circulating oral microbial toxins, and (iii) metastatic inflammation caused by immunological injury induced by oral microorganisms (43).

The diagnostic possibilities of periodontal diseases are based on knowledge of their aetiology and pathogenesis. In periodontitis methods to date have focused primarily on the protein expression of inflammation and tissue destruction and oral microbiology. Molecular biochemistry has also brought more recent knowledge about this disease (see Fig. 1).

An element of dentistry is the diagnosis of periodontal disease and monitoring of traditional parameters, which includes the probing depth (PD) of the gingival sulcus, the gingival index (GI), clinical evaluation of insertion (clinical attachment level-CAL), gingival recession (GR), bleeding on probing (BOP), the plaque index (PI) and radiodiagnostical analysis. These parameters, however, have their limitations in fact, disadvantages. These include, in particular:

1. these diagnostic parameters are an excellent indicator of history of the disease, however, if we do not have standardized long-term measurements, only limited opportunities for determining the further development of the disease is provided;

2. the damage must be significant in order for these parameters to provide information about the severity of the disease.

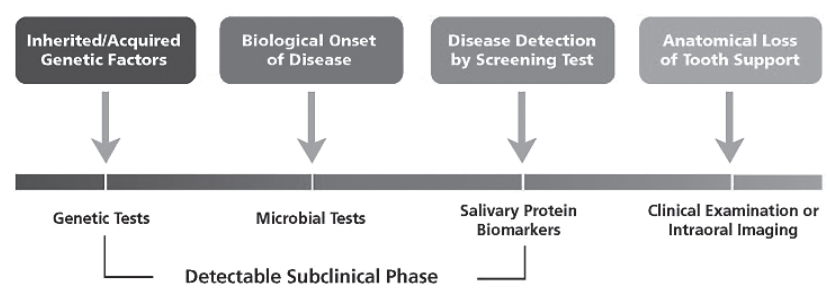

Fig. 1: Timeline of periodontal disease progression.

(Source: Giannobile WV. Salivary diagnostics for periodontal disease. JADA 2012; 143(suppl 10): 6S-11S.) 
As a result of these disadvantages, laboratory molecular-biochemical processes are increasingly used for the needs of early diagnosis and predicting the worsening of the disease with emphasis on their usefulness in routine outpatient practice.

\section{Biological material}

The use of conventional biological materials, such as whole blood (serum/plasma) and urine, are disadvantageous, because of the initially local nature of the disease. A specific marker characteristic only for periodontal tissues has not yet been found. Based on these facts, the most appropriate biological materials are therefore considered to be gingival crevicular fluid and saliva.

\section{Gingival crevicular fluid}

Sulcus fluid (GCF - Gingival crevicular fluid) penetrates into the gingival sulcus from the gingival tissue. It arises as a plasma transudate or more often as a result of inflammatory exudation. Its physiological function is to clean the gingival sulcus; penetrated proteins facilitate the adherence of the free gingiva to the tooth, and the fluid also exhibits antimicrobial properties. In a healthy periodontal tissue it forms in a minimum amount; however, under pathological circumstances formation harply increases (up to thirty-fold).

The main advantage of using it is the site specificity, significantly visible in orthodontic therapy (20). In addition, GCF may be collected as peri-implant sulcular fluid (PISF) from the gingival cuff that surrounds a dental implant (3). The most commonly used devices for collection are strips of filter paper and micropipettes.

Methods for measuring only the volume of the fluid are nowadays considered insufficient and have been replaced with the composition analyses, which can identify individual proteins of inflammatory and immune responses, proteinase inhibitors, hydrolytic enzymes, intracellular proteins, construction proteins of the cytoskeleton and apoptotic and signalling proteins $(6,40)$. Based on the above-mentioned analyses, it is possible to determine the current level of periodontal damage, and the future course of the disease can be anticipated. Although analysis of gingival crevicular fluid helps provide an explanation of the inflammatory response in periodontitis, multiple collection of samples with the assistance of traditional filter papers is currently considered to be impractical in clinical practice $(22,27)$. In recent years gingival crevicular fluid been revived as an excellent indicator of the current local state of the periodontal tissue during the testing of local application of biodegradable nanoparticles of drugs (50).

\section{Saliva}

Biological material such as saliva, given its natural presence in the oral cavity, is also available for research on potential markers of periodontitis. It has already been used in diagnostics for a number of systemic diseases, and the use of saliva is also commercially available through many tests. Saliva is formed by the mixing of liquid products of the large and small salivary glands and also includes components of gingival crevicular exudate, expectorated bronchial secretions, serum, blood cells from the oral micro-wounds, bacteria and their products, viruses, fungi, peeled epithelial cells and food particles (10). The main advantages of saliva as a biological material are the painless, non-invasive collection, the ability to repeat sampling and the easy transport and storage. In addition, the collection of saliva does not require a trained person; delivery is safer for staff and saliva is considered to be a "real-time" material. This attribute makes saliva suitable for monitoring children, the elderly and non-cooperative patients and not only in circumstances where the collection of blood or urine is not possible. The most appropriate method for collecting whole (glandular non-specific) saliva is considered to be the drainage method (drip off the bottom lip, spitting directly into the container) (37).

\section{Potential biomarkers of periodontitis in saliva}

The main groups of potential markers for periodontitis include: inflammatory markers, markers of connective tissue destruction and bone remodelling markers.

\section{Markers of inflammation}

Inflammation is often seen as something noxious for the body, but its current definition is completely the opposite. Every case of inflammation has a primary defence mission. This can manifest itself by an acute cell response, and if the complaint persists for a long enough time, it may turn into a chronic response. Objectively identifiable mediators are used as inflammatory biomarkers. The most commonly used markers for detection in periodontitis are mainly $\beta$-glucuronidase (GUS), C-reactive protein (CRP), interleukin-1 (IL-1), interleukin-6 (IL-6), macrophage inflammatory protein $1 \alpha$ (MIP-1 $\alpha)$ and tumour necrosis factor (TNF) (36). The level of $\beta$-glucuronidase, which signals the influx of neutrophils, was confirmed in correlation with the increasing severity of periodontitis (28). Similarly, this correlation was confirmed in IL-1 $\beta$, an important pro-inflammatory cytokine that is the predominant form of periodontitis (two forms: IL-1 alpha and IL-1 beta) and also in TNF- $\alpha(7,8,48)$. The newly identified anti-inflammatory interleukin IL-35, which is a member of the IL-12 family, is secreted by regulatory T-cells and suppresses the inflammatory response of immune cells. In one study it had significantly the highest level in saliva in a healthy group compared with groups with periodontal disease (gingivitis, chronic periodontitis), which shows its important role in the suppression of periodontal inflammation and maintaining of periodontal health (25). 


\section{Markers of connective tissue breakdown}

The breakdown of connective tissue is responsible for pathogenesis of chronic inflammatory conditions and this also occurs in periodontitis. Matrix degradation is initiated by proteases produced locally at the site of inflammation and is balanced with their inhibitors. The degree of balance appears to be decisive for the progression of chronic periodontitis. The most commonly assessed are $\alpha 2$-macroglobulin, matrix metalloproteinases (MMPs), tissue inhibitors of metalloproteinases (TIMPs) and alanine aminotransferase (ALT) levels. Levels of $\alpha 2$-macroglobulin, which is intended for the inactivation of different proteases including metalloproteinases and collagenase, are recorded as reduced in adult patients with periodontal disease, which under these conditions shows an imbalance between proteases and their inhibitors (1). This idea is supported by findings of an increase in tissue inhibitors in saliva after conventional treatment of periodontitis (13). The most important proteolytic enzymes are the matrix metalloproteinases MMP-8 (collagenase-2) and MMP-9 (gelatinase), a significant increase of which was confirmed in the saliva of patients with periodontitis. Monitoring of the level of MMP-8 in particular has potential for clinical use $(41,33)$.

\section{Markers of bone remodelling}

Salivary markers of alveolar bone remodelling (bone resorption/bone formation) are still not described as being as good as the biomarkers of the two above-mentioned groups. And the episodic nature of this process, which occurs during the progression of periodontal disease (predominantly resorption), also participates in this. This subject still requires longitudinal studies, but such research is much more expensive than cross-sectional studies. Longitudinal studies would be especially suitable for patients with aggressive periodontitis (36). The main problem with these biomarkers lies in their extremely low concentrations at the time of remission and at the time of exacerbation of premature degradation in saliva (8). The most important such biomarkers are alkaline phosphatase (ALP), C-terminal telopeptide (carboxy-terminal collagen crosslinks or CTX) and a recent receptor activator of nuclear factor kappa-B ligand (receptor activator of NF- $\kappa$ B or RANKL) and osteoprotegerin (OPG). Consequently, the historical performance of studies is often contradictory to longitudinal studies, and larger groups of patients could provide beneficial data on these bone markers in the context of periodontal disease.

\section{Creating of diagnostic panel for periodontitis}

In general, one could say that accurate diagnostic information about this disease can be obtained if a combination of appropriate biomarkers with the necessary sensitivity and specificity is created. For the purpose of threshold determination for periodontitis, several combinations of parameters were used; in one case the combination of MMP- 8 and IL-1 $\beta$ showed an association with a significantly higher risk for periodontal disease. Combinations of these parameters also more often exhibit a positive predictive value for confirmation of the disease $(36,8)$.

\section{Oral microbiology}

An inseparable part of laboratory diagnosis of periodontal disease is indisputably microbiological diagnosis. The oral cavity has two special features with regard to microorganisms: it includes various micro environments which are contained in one complex, and microorganisms do not live as single species, but in colonies. Characteristic is the colonization of opportunistic microorganisms, which un-

Tab.1: Typical prevalent bacterial composition in selected cases of periodontitis.

(Source: Lamont RJ., Jenkinson HF. Oral microbiology at Glance. 2010, Wiley-Blackwell - modified.)

\begin{tabular}{|c|c|c|c|}
\hline Healthy periodont & Gingivitis & Chronic periodontitis & Agressive periodontitis \\
\hline $\begin{array}{l}\mathbf{G}^{+} \\
\text {S. oralis, mitis, S. gordo- } \\
\text { nii, sanguinis, Actinomyces } \\
\text { gerencseriae, Actinomyces } \\
\text { naeslundi }\end{array}$ & $\begin{array}{l}\mathbf{G}^{+} \\
\text {Lactobacillus species, } \\
\text { Actinomyces naeslundii, } \\
\text { Peptostreptococcus micros, } \\
\text { Streptococcus onginosus, } \\
\text { Fusobacterium nucleatum }\end{array}$ & $\begin{array}{l}\mathbf{G}^{+} \\
\text {Eubacterium brachy, } \\
\text { Eubacterium nodatum, } \\
\text { Peptostreptococcus stomatis }\end{array}$ & \\
\hline $\begin{array}{l}\mathbf{G}^{-} \\
\text {Fusobacterium species, } \\
\text { Prevotella nigrescens, } \\
\text { Veillonella species }\end{array}$ & $\begin{array}{l}\mathbf{G}^{-} \\
\text {Prevotella intermedia, } \\
\text { Fusobacterium nucleatum, } \\
\text { Campylobacter species, } \\
\text { Haemophilus species, } \\
\text { Selenomonas species, } \\
\text { Treponema species }\end{array}$ & $\begin{array}{l}\mathbf{G}^{-} \\
\text {Porphyromonas gingivalis, } \\
\text { Tannerella forsythia, } \\
\text { Treponema denticola, } \\
\text { Campylobacter rectus, } \\
\text { Prevotella intermedia, } \\
\text { Fusobacterium nucleatum }\end{array}$ & $\begin{array}{l}\mathbf{G}^{-} \\
\text {Actinobacillus actinomycetem- } \\
\text { comitans, Porphyromonas } \\
\text { gingivalis, Tannerella } \\
\text { forsythia, Prevotella inter- } \\
\text { media, Prevotella nigrescens, } \\
\text { Fusobacterium nucleatum, } \\
\text { Campylobacter rectus }\end{array}$ \\
\hline
\end{tabular}


der certain conditions are capable of causing disease (26). Equally characteristic is the presence of certain bacterial species at certain stages of periodontal diseases (see Table 1). Microorganisms, especially in the subgingival area, can activate a cascade of defence mechanisms associated with the production of factors causing inflammation and tissue destruction.

\section{Options for microbiological diagnosis of periodontal diseases}

The culturing of oral bacteria is the gold standard that is generally used, but this method has its pitfalls, especially tracking the presence of anaerobic species. Their culture result is often underestimated and only living bacteria can be culture, of course; their transport is also difficult, and many require specific conditions for growth. Finally, this method also requires special laboratory equipment and experienced staff and is very time consuming. All of these drawbacks led to the introduction of PCR (Polymerase Chain Reaction) for the identification of periodontal pathogens by their species-specific DNA sequence (42).

\section{Qualitative methods}

Qualitative methods (PCR based methods, enzymatic methods) are useful for confirming the presence of a certain type of bacteria, but say nothing about their quantity. They should be used for monitoring changes in the composition of bacteria after treatment in comparison with situation before treatment, which would help to assess its effectiveness (52).

\section{Quantitative methods}

Real-time PCR is used for measuring the amount of DNA. It can be used for both qualitative and quantitative analysis. This method has also been applied to measure the number of bacteria in periodontal diseases in samples such as saliva and GCF. The results showed, for example, a significant correlation between the number of bacteria and a deep pocket. Specifically, the number of Porphyromonas gingivalis increased to ten-times each growing millimetre of pocket depth $(21,52)$.

\section{Molecular diagnosis in periodontology}

Destructive periodontal diseases in the generally accepted view are initiated by changes in the bacterial flora, which triggers an immune response in susceptible individuals. This immune response is dependent on the nature and virulence of the pathogen. However, in most cases, the presence of a particular microorganism alone is not sufficient to initiate the disease. These findings suggest that environmental and genetic factors may influence the development of the disease. Specific genetic mutations that are responsible for causing periodontal diseases are rare and fortunately do not characterize the most common forms of periodontitis. Family cumulation of this disease is uncommon, and finding it can mean the impact of genetic predisposition but also exposure to the same external environmental influences (38). According to the studies conducted in twins, it appears that less than half of such variability is accounted for by genetic variability (35). Currently, studies are focusing on genetic polymorphisms of candidate genes associated with disease susceptibility (45).

\section{Gene polymorphism in periodontitis}

Genetic polymorphism is a form of a gene (allelic variant) which is found in an amount of at least $1 \%$ of a population, which is the border between a polymorphism and a mutation, while a mutation is referred to as an allele frequency of less than $1 \%$ in the population (32). Genetic polymorphism is more frequent in the population than mutation, and correlation between genetic polymorphism and disease is generally weaker than the functional relationship between mutation and disease (47).

The most studied polymorphisms in relation to periodontitis include the genes for cytokines (particularly IL-1 and IL-6), the genes for the receptors (Fc gamma receptor, Toll-like receptor), the genes for the RANK/RANKL/ OPG and genes that encode enzymes (cathepsin C, matrix metalloproteinases, cyclooxygenase 2, myeloperoxidase, $\mathrm{N}$-acetyl-transferase). The results to date are not clear, because they have not been obtained from a larger groups of patients, but some results are already known, e.g. the polymorphisms in Fc receptor III b, which serves (Fc receptor III b) first and foremost as a binding site for IgG on phagocytic cells, affects their mutual binding affinity and some of which are considered to be susceptible to periodontal disease. It has two polymorphisms, referred to as NA1 and NA2. The Fc $\gamma$ RIIIb-NA2 allele and NA2/NA2 genotype occurred more frequently in controls and NA2/NA2 again more frequently in patients with the generalized aggressive form of periodontitis (GAgP) (16). The distribution of genotypes was significantly different among different races, and it seems that the relationship between Fc $\gamma \mathrm{R}$ polymorphisms and periodontal disease is associated with racial affiliation $(9,23,49)$. Homozygous carriers of the polymorphism of a myeloperoxidase $(-463 \mathrm{G} / \mathrm{G})$ are at increased risk of periodontitis they are at the same time smokers (34). The situation is reversed e.g. in one polymorphism in the gene for COX 2 (cyclooxygenasis 2 ). Representation mostly covers a single nucleotide polymorphism $(-756 \mathrm{G} \rightarrow \mathrm{C})$ and its protective effect in particular from aggressive periodontitis (15).

Several studies of the relationship between gene polymorphisms (for example matrix metalloproteinase 8, toll-like receptor 4, apolipoprotein E, interleukin 8) in patients with periodontitis were performed also in Czech population $(4,5,18,19)$.

To date, major gene mutations, which result in the periodontitis phenotype in otherwise systemically healthy 
individuals, have not been identified and no specific genetic risk factor for the disease has been identified. The aim of the test is to determine several relatively common high-risk polymorphisms, that may mean cumulative high susceptibility genetic profile (47).

\section{Epigenetic changes in periodontal diseases}

In term of genetics, not only a mutation or polymorphism in a gene impacts an individual. Epigenetics is the science that deals with the study of changes in gene expression, which do not involve changes in the DNA sequence. Epigenetics is applied, for example, in the chemical modification of DNA and its proteins by blocking the binding of transcription factors, including the modifications of histones and DNA methylation (2). In subjects with a severe form of periodontitis hypomethylation of the gene for IL-6 was observed, which incites its increased expression in tissue affected by inflammation. Of interest are the speculations that long-standing inflammation and bacterial infection can also result in the methylation of DNA, which inactivates the suppression of cytokine signalling and contributes to the exaggeration of the inflammation (46). The number of studies on epigenetic changes in periodontitis is rapidly increasing (29)

\section{Genetic testing}

For the detection of polymorphisms PCR-RFLP (Restriction Fragment Length Polymorphism) is frequently used, which is based on the existence of restriction endonucleases that cleave the DNA polynucleotide chain within the area of the phosphodiester bonds in certain specific sequences. However, today many other methods for detecting polymorphisms and modification of DNA are known. For example, a genetic test is available for general public which is focused on severe chronic periodontitis (for IL-1 and IL-6 genetic variations). It focuses on specific polymorphisms that are associated with the disease in the respective country and by race (11).

\section{Recent trends in the diagnosis of periodontal diseases}

The transfer of possibilities for diagnostics of periodontal diseases that are easily incorporated into routine dental practice could mean earlier, simpler and more intensive treatment, which would likely bring even more cost-effective oral healthcare. Patients would benefit from tests carried out at home, according to the demands of conducting them, and sold without a prescription. The introduction of such measures would likely increase personal interest in treatment and overall compliance with the proposed therapeutic recommendations.

For this purpose devices that are generally used in the place where the patient is located should serve and perma- nently allocated space and tests performed outside the central laboratory should not be required for them. These tests will reduce the cost of transportation, packaging, handling, storage and tracking samples to the central laboratory, thus reducing the likelihood of sample contamination or sample confusion, loss or degradation (30). The device should combine and use modern nanomaterials, microfluidic engineering and microelectronics for the practical creation of miniature sensors. Importantly, the results of the use of these miniature sensors thus far correlate with standard methods used at present (36). In terms of using an oral fluid, several tests are now commercially available, for example, that allow detection of antibodies to HIV, steroid hormones, alcohol and drugs as well as forensic and genetic analysis (14).

Nano-biochips, which integrate various laboratory procedures in a single cartridge (device), are currently considered to be the most appropriate for this type of diagnosis. A saliva sample (100-300 microlitres) or a drop of blood is sufficient for the diagnosis. A network of liquid components ensures complete transfer and processing of salivary samples for multiple analyses in order to provide quantitative and qualitative information on the target biomarkers of disease. Starting the analysis is automatic, without the need for human intervention, and the internal flexibility of the software allows further modifications. The biochips used are disposed of as solid organic waste (36). The principle of the analysis may like that for immunoassays, the most common of which are the LF strip and the ELISA method.

More complicated was the problem of using biochips in molecular diagnostics. It is now possible to carry out analysis of a nucleic acid in a continuous flow simultaneously with the necessary temperature control (see Fig. 2). All the reagents are present on the chip; the temperature is controlled

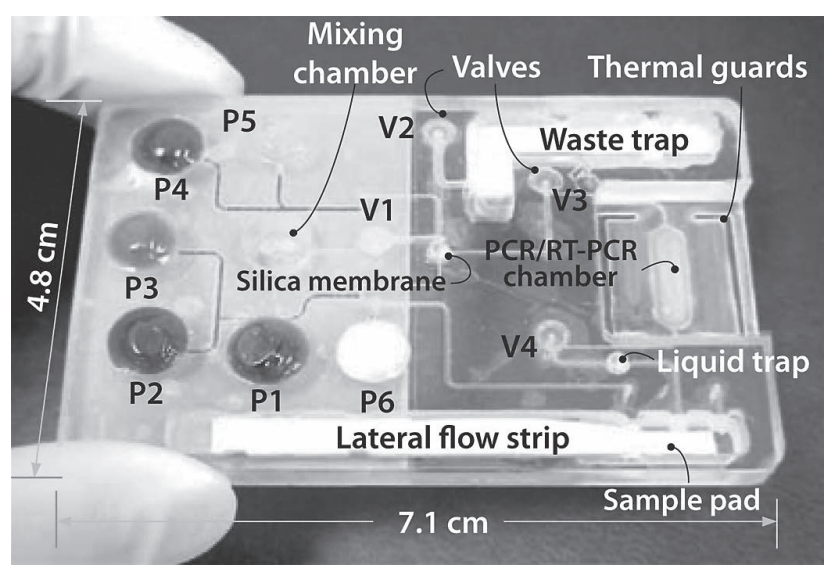

Fig. 2: Disposable cartridge for PCR.

The plastic case includes a network for microfluidic lysis, isolation of nucleic acids on a solid phase, PCR and PCR-detection products labelled with phosphorus on an LF strip.

(Source: Hart RW, Mauk MG, Liu C, Qiu X, Thompson JA, Chen D, Malamud D, Abrams WR, Bau HH. Point-of-care oral-based diagnostics. Oral Dis 2011 Nov; 17(8): 745-52.) 
by thermal chamber, and the individual reagents are placed in a wax which melts during the initial heating and reagents are hydrated in the kit. The amplification process can be observed in real time, when using an appropriate colour, e.g. SYBR Green, a fluorescent dye that emits light only when the intercalate with double-stranded DNA (17). A modified standard PCR method, which is called the LAMP (Loop-mediated isothermal amplification), may be at approximately the same temperature to carry out the amplification (multiplication of the DNA) in a short time (approx. 1 hour), and the result is visible to the naked eye.

\section{Perspective of PoCT in periodontics}

With the convergence of microfluidic techniques and diagnosis of oral fluids, it would be possible to diagnose and monitor a patient with on-site testing, in an outpatient department, at home, or even in remote areas. The purpose is to support individualized treatment, or "treatment tailored for each patient". And oral fluids (saliva, GCF) are ideal for such measurements. Point-of-care testing (PoCT) may be of particular interest in the dental community because patients usually visit a dentist more frequently than a general physician (39).

\section{Conclusion}

Oral health does not mean only an attractive smile; the term encompasses a comprehensive view of the oral cavity under physiological conditions. Today we know that pathologies in this area can affect the overall condition of the body, and the connection is evaluated in the context of many systemic diseases.

Periodontal diseases are still living issue. Although periodontal diagnostic testing initially served to delimit patients at higher risk for developing this disease, the future of these laboratory tests is now extended to patients at risk of developing systemic diseases caused by periodontitis, and if this risk is confirmed, the disease may be reduced by effective treatment. Finding suitable markers, whether for early diagnosis, exacerbation or other consequences of this disease would mean not only a reduction of the suffering of more than tens of millions of people around the world, but also cut the cost of their treatment.

Currently, saliva has come to the forefront as a biological material. Equally high hopes are placed on molecular diagnostics. Some tests are already available commercially, but their acceptance in medical practice is slow, in part due to the lack of treatment algorithms that would give clear guidance for their use in the provision of health care. Scientists see the future of complex scientific research in this area in a comprehensive approach to the examination of biological materials using equipment that would be able to simply, specifically and sensitively investigate suitable parameters, even in outpatient clinic, and perhaps include additional screening parameters for other diseases.
The question is whether we are separated from such results by a great deal of research work or only a few small steps.

\section{References}

1. Aurer A, Jorgic-Srdjak K, Plancak D, Stavljenic-Rukavina A, Aurer-Kozelj J. Proinflammatory factors in saliva as possible markers for periodontal disease. Coll Antropol 2005; 29(2): 435-439.

2. Backdahl L, Bushell A, Beck S. Inflammatory signalling as mediator of epigenetic modulation in tissue-specific chronic inflammation. Int J Biochem Cell Biol 2009; 41(1): 176-84.

3. Bhardwaj S, Prabhuji ML. Comparative volumetric and clinical evaluation of peri-implant sulcular fluid and gingival crevicular fluid. J Periodontal Implant Sci 2013; 43(5): 233-42.

4. Borilova Linhartova P, Bartova J, Poskerova H, et al. Apolipoprotein E gene polymorphisms in relation to chronic periodontitis, periodontopathic bacteria, and lipid levels. Arch Oral Biol 2015 Mar; 60(3): 456-62.

5. Borilova Linhartova P, Vokurka J, et al. Haplotype analysis of interleukin-8 gene polymorphisms in chronic and aggressive periodontitis. Mediators Inflamm 2013: 342-351.

6. Carneiro LG, Venuleo C, Oppenheim FG, Salih E. Proteome data set of human gingival crevicular fluid from healthy periodontium sites by multidimensional protein separation and mass spectrometry. Journal of Periodontal Research 2012; 47: $248-262$.

7. Fine DH, Markowitz K, Furgang D, et al. Macrophage inflammatory protein-1a: a salivary biomarker of bone loss in a longitudinal cohort study of children at risk for aggressive periodontal disease? J. Periodontol 2009; 80(1): 106-113.

8. Frodge BD, Ebersole JL, Kryscio RJ, Thomas MV, Miller CS. Bone remodeling biomarkers of periodontal disease in saliva. J. Periodontol 2008; 79(10): 1913-1919.

9. Fu Y, Korostoff JM, Fine DH, Wilson ME. Fc $\gamma$ receptor genes as risk markers for localized aggressive periodontitis in African-Americans. J Periodontol 2002; 73: $517-523$.

10. Giannobile WV, Beikler T, Kinney JS, Ramseier CA, Morelli T, Wong DT. Saliva as a diagnostic tool for periodontal disease:current state and future directions. Periodontol 2000 2009; 50: 52-64.

11. Giannobile WV, Braun TM, Caplis AK, Doucette-Stamm L, Duff GW, Kornman KS. 2013. Patient Stratification for Preventive Care in Dentistry. J Dent Res 92(8): 694-701.

12. Giannobile WV. Salivary diagnostics for periodontal disease. JADA 2012; 143(suppl 10): 6S-11S.

13. Gorska R, Nedzi-Gora M. The effects of the initial treatment phase and of adjunctive low-dose doxycycline therapy on clinical parameters and MMP-8, MMP-9, and TIMP-1 levels in the saliva and peripheral blood of patients with chronic periodontitis. Arch Immunol Ther Exp 2006; 54(6): 419-426.

14. Hart RW, Mauk MG, Liu C, et al. Point-of-care oral-based diagnostics. Oral Dis 2011; 17(8): 745-52.

15. Ho YP, Lin YC, Yang YH, Ho KY, Wu YM, Tsai CC. Cyclooxygenase-2 Gene-765 single nucleotide polymorphism as a protective factor against periodontitis in Taiwanese. J Clin Periodontol 2008; 35: 1-8.

16. Chai L, Song YQ, Leung WK. Genetic polymorphism studies in periodontitis and Fc $\gamma$ receptors. J Periodontal Res 2012 Jun; 47(3): 273-85.

17. Chen D, Mauk M, Qiu X, et al. An integrated, selfcontained microfluidic cassette for isolation, amplification, and detection of nucleic acids. Biomed Microdevices 2010; 12(4): 705-19.

18. Izakovicova Holla L, Buckova D, Fassmann A, Roubalikova L, Vanek J. Lack of association between chronic periodontitis and the Toll-like receptor 4 gene polymorphisms in a Czech population. J Periodontal Res 2007 Aug; 42(4): 340-4.

19. Izakovicova Holla L, Hrdlickova B, Vokurka J, Fassmann A. Matrix metalloproteinase 8 (MMP8) gene polymorphisms in chronic periodontitis. Arch Oral Biol 2012 Feb; 57(2): 188-96.

20. Kapoor P, Kharbanda OP, Monga N, Miglani R, Kapila S. Effect of orthodontic forces on cytokine and receptor levels in gingival crevicular fluid: a systematic review. Prog Orthod 2014; 15: 65.

21. Kawada M, Yoshida A, Suzuki N, et al. Prevalence of Porphyromonas gingivalis in relation to periodontal status assessed by real-time PCR. Oral Microbiology and Immunology 2004; 19(5): 289-292.

22. Khiste SV. Critical analysis of biomarkers in the current periodontal practice. J Indian Soc Periodontol 2011; 15(2): 104-10.

23. Kobayashi T, Ito S, Kuroda T, et al. The Interleukin-1 and Fc $\gamma$ Receptor gene polymorphisms in Japanese patients with rheumatoid arthritis and periodontitis. J Periodontol 2007; 78, 2311-2318.

24. König J, Holtfreter B, Kocher T. Periodontal health in Europe: future trends based on treatment needs and the provision of periodontal services - position paper 1 . Eur J Dent Educ 2010; 14: 4-24. 
25. Köseoğlu S, Sağlam M, Pekbağrıyanık T, Savran L, Sütcü R, Level of Interleukin-35 in Gingival Crevicular Fluid, Saliva and Plasma in Periodontal Disease and Health. J Periodontol 2015 Mar; 19: 1-12.

26. Lamont RJ, Jenkinson HF. Oral Microbiology at a Glance. Oxford: Wiley-Blackwell 2010.

27. Lamster IB, Ahlo JK. Analysis of gingival crevicular fluid as applied to the diagnosis of oral and systemic diseases. Ann N Y Acad Sci 2007; 1098: 216-29.

28. Lamster IB, Kaufman E, Grbic JT, Winston LJ, Singer RE. $\beta$-glucuronidase activity in saliva: relationship to clinical periodontal parameters. J. Periodontol 2003; 74(3): 353-359.

29. Lavu V, Venkatesan V, Rao SR. The epigenetic paradigm in periodontitis pathogenesis, J Indian Soc Periodontol 2015; 19(2): 142-149.

30. Lee-Lewandrowski E, Lewandrowski K. Perspectives on cost and outcomes for point of-care testing. Clin Lab Med 2009; 29: 479-489.

31. Li X, Kolltveit KM, Tronstad L, Olsen I. Systemic diseases caused by oral infection. Clin Microbiol Rev 2000 Oct; 13(4): 547-58.

32. Loos BG, Tjoa S. Host derived diagnostic markers for periodontitis: do they exist in gingival crevice fluid? Periodontology 2000 2005; 39: 53-72.

33. Marcaccini AM, Meschiari CA, Zuardi LR, et al. Gingival crevicular fluid levels of MMP-8, MMP-9, TIMP-2, and MPO decrease after periodontal therapy. J Clin Periodontol 2010; 37(2): 180-90.

34. Miesel P, Siegeund A, Grimm R, et al. The interleukin-1 polymorphism, smoking and the risk of periodontal disease in the population based SHIP study. J Dent Re 2003; 82: 189-93.

35. Michalowicz BS, Diehl SR, Gunsolley JC, et al. Evidence of a substantial genetic basis for risk of adult periodontitis. J Periodontol 2000; 71(11): 1699-707.

36. Miller CS, Foley JD, Bailey AL, et al. Current developments in salivary diagnostics. Biomark Med 2010; 4(1): 171-89.

37. Navazesh M. Methods for collecting saliva. Ann N Y Acad Sci 1993; 694: $72-7$.

38. Nibali L, Donos N, Brett PM, Parkar M, Ellinas T, Llorente M. A familial analysis of aggressive periodontitis - clinical and genetic findings. J Periodontal Res 2008 43(6): 627-34.

39. Paegel BM, Joyce GF. Microfluidic compartmentalized directed evolution. Chem Biol 2010; 17: 717-724.
40. Pisano E, Cabras T, Montaldo C, et al. Peptides of human gingival crevicular fluid determined by HPLC-ESI-MS. Eur J Oral Sci 2005; 113(6): 462-8.

41. Romero AM, Mastromatteo-Alberga P, Escalona L, Correnti M. MMP-3 and MMP-8 levels in patients with chronic periodontitis before and after nonsurgical periodontal therapy. Invest Clin 2013; 54(2): 138-48.

42. Sanz M, Lau L, Herrera D, Morrilo JM, Silva A. Methods of detection of Actinobacillus actinomycetemcomitans, Porphyromonas gingivalis and Tannerella forsythensis in periodontal microbiology, with special emphasis on advanced molecular techniques: a review. Journal of Clinical Periodontology 2004; 31, 1034-1047.

43. Seymour GJ, Ford PJ, Cullinan MP, Leishman S, Yamazaki K. Relationship between periodontal infections and systemic disease. Clin Microbiol Infect 2007 Oct; 13 Suppl 4: 3-10

44. Shiau HJ, Reynolds MA. Sex differences in destructive periodontal disease: exploring the biologic basis. J Periodontol 2010; 81(11): 1505-17.

45. Schafer AS, Jepsen S, Loos BG. Periodontal genetics: a decade of genetic association studies mandates better study designs. J Clin Periodontol 2011; 38(2): 103-7.

46. Stenvinkel P, Karimi M, Johansson S, Axelsson J, Suliman M, Lindholm B. Impact of inflammation on epigenetic DNA methylation - a novel risk factor for cardiovascular disease? J Intern Med 2007; 261(5): 488-99.

47. Tarannum F, Faizuddin M. Effect of gene polymorphisms on periodontal diseases. Indian J Hum Genet 2012; 18(1): 9-19.

48. Tobon-Arroyave SI, Jaramillo-Gonzalez PE, Isaza-Guzman DM. 2008. Correlation between salivary IL-1 $\beta$ levels and periodontal clinical status. Arch Oral Biol 53(4): 346-352.

49. Yamamoto K, Kobayashi T, Grossi S, et al. Associationof Fcy receptor IIa genotype with chronic periodontitis in Caucasians. J Periodontol 2004; 75: 517-522.

50. Yao W, Xu P, Pang Z, et al. Local delivery of minocycline-loaded PEG-PLA nanoparticles for the enhanced treatment of periodontitis in dogs. Int J Nanomedicine 2014; 9: 3963-3970.

51. Yoshida A. Ansai T. Microbiological diagnosis for periodontal disease. Periodontal diseases - A clinician's guide, 2012.

52. Yoshida A, Kawada M, Suzuki, et al. TaqMan real-time polymerase chain reaction assay for the correlation of Treponema denticola numbers with the severity of periodontal disease. Oral Microbiology and Immunology 2004; 19(3): 196-200.

Received: $15 / 05 / 2015$

Accepted in revised form: 22/02/2016

\section{Corresponding author:}

Beáta Bolerázska, Department of Medical and Clinical Biochemistry Faculty of Medicine, P. J. Šafárik University in Košice, Trieda SNP 1, 04011 Košice, Slovakia; e-mail: beata@moly.sk 